\title{
Rotational excitation and de-excitation of PN molecules by He atoms
}

\author{
R. Toboła ${ }^{1}$, J. Kłos ${ }^{2}$, F. Lique ${ }^{2}$, G. Chałasiński ${ }^{1,3}$, and M. H. Alexander ${ }^{2}$ \\ 1 Faculty of Chemistry, University of Warsaw, ul. Pasteura 1, 02-093 Warszawa, Poland \\ 2 Department of Chemistry and Biochemistry, University of Maryland, College Park, MD 20742, USA \\ 3 Department of Chemistry, Oakland University, Rochester MI 48309, USA \\ e-mail: francois. lique@obspm. fr
}

Received 22 February 2007 / Accepted 14 March 2007

\begin{abstract}
Context. Observations of molecular emission at millimeter and infrared wavelengths, supplemented by careful and detailed modeling, are powerful tools for investigating the physical and chemical conditions of astrophysical objects. Modeling of molecular emission requires the calculation of rates for excitation by collisions with the most abundant species.

Aims. The present paper focuses on the calculation of rate coefficients for rotational excitation of the PN molecule in its ground vibrational state in collision with $\mathrm{He}$, based on a new two-dimensional potential energy surface from highly correlated ab initio calculations.

Methods. Calculations of pure rotational (de)excitation of PN by He were performed for the first rotational levels using the essentially exact close-coupling method for the lower levels and the coupled-states approximation for higher levels.

Results. Cross sections for transitions among the 31 first rotational levels of PN were calculated for kinetic energies up to $2500 \mathrm{~cm}^{-1}$. These cross sections were used to determine collisional rate constants for temperatures ranging from $5 \mathrm{~K}$ to $300 \mathrm{~K}$. A propensity for even $\Delta j$ transitions is observed, which reflects the nearly symmetric character of the PN-He potential energy surface.
\end{abstract}

Key words. ISM: molecules - molecular data - molecular processes

\section{Introduction}

The first interstellar detection of the PN molecule was reported more than 20 years ago by Turner \& Bally (1987) and Ziurys (1987). This was the first example of a phosphoruscontaining species to be seen in interstellar molecular clouds. The PN molecule has been identified in Ori(KL), W51M, and SgrB2, by observations of the $j=2 \rightarrow 1, j=3 \rightarrow 2$ and $j=5 \rightarrow 4$ rotational transitions. Because it has a dipole moment, $\mathrm{PN}$ is a good candidate for radioastronomy. The PN molecule has been frequently observed (Turner et al. 1990; Turner 1991; Schilke et al. 1997; Tercero \& Cernicharo 2007), so far only in star-forming regions. The chemistry of PN has been the object of several studies (Millar 1991; Charnley \& Millar 1994). The modeling of PN molecular emissions requires excitation calculations based on radiative rates as well as rate coefficients for collisions with the most abundant interstellar species, namely $\mathrm{He}$ and $\mathrm{H}_{2}$.

Recent studies (Lique et al. 2006; Daniel et al. 2007) have shown that modeling studies based on inaccurate collisional excitation rates can lead to important errors in the determination of molecular abundances in molecular clouds. As far as we know, no collisional rates exist for the PN molecule. The focus of this paper is the theoretical determination of accurate PN collisional rate coefficients with the interstellar species $\mathrm{He}$ and $\mathrm{H}_{2}$, specifically for temperatures from 5 to $300 \mathrm{~K}$. To this end we describe close-coupling and coupled-states quantum scattering calculations based, on a potential energy surface determined using highly-correlated coupled-cluster methods.
The Herschel satellite of the European Space Agency (ESA) is expected to provide, in the next few years, high spectral resolution observations of molecular emission in the submillimeter and far-IR domains. Given the potential sensitivity of the Herschel instrument, we anticipate, specifically, that PN lines involving high- $j$ levels will be observed with high signal to noise ratios. Consequently, we will consider here transitions between the lowest 31 rotational levels of PN. We expect that the present results will be relevant for interpreting the data that both Herschel as well as ALMA will provide over the next few years.

The paper is organized as follows: Sect. 2 describes the ab initio calculation of the potential energy surface. Section 3 then contains a rapid description of the scattering calculations. In Sect. 4 we present and discuss our results.

\section{Potential energy surface}

The ground electronic state of the very weakly bound $\mathrm{PN}-\mathrm{He}$ system is a singlet with $A^{\prime}$ refection symmetry. The $\mathrm{PN}-\mathrm{He}$ "rigid rotor" potential energy surface (PES) is described by the two Jacobi coordinates $R$, the distance from the center of mass of PN to the He atom, and $\theta$, the angle between $\boldsymbol{R}$ and the PN bond axis $\boldsymbol{r}$, with $\theta=0$ corresponding to colinear HeNP. The PN bond distance $r$ was frozen at its experimental equilibrium value $r_{\mathrm{e}}=2.81733$ bohr (Huber \& Herzberg 1979). As demonstrated by Lique et al. (2007) for the CS-He system, for non-hydride diatomic molecules two-dimensional PESs calculated for a frozen bond distance or obtained from full 3D PESs 


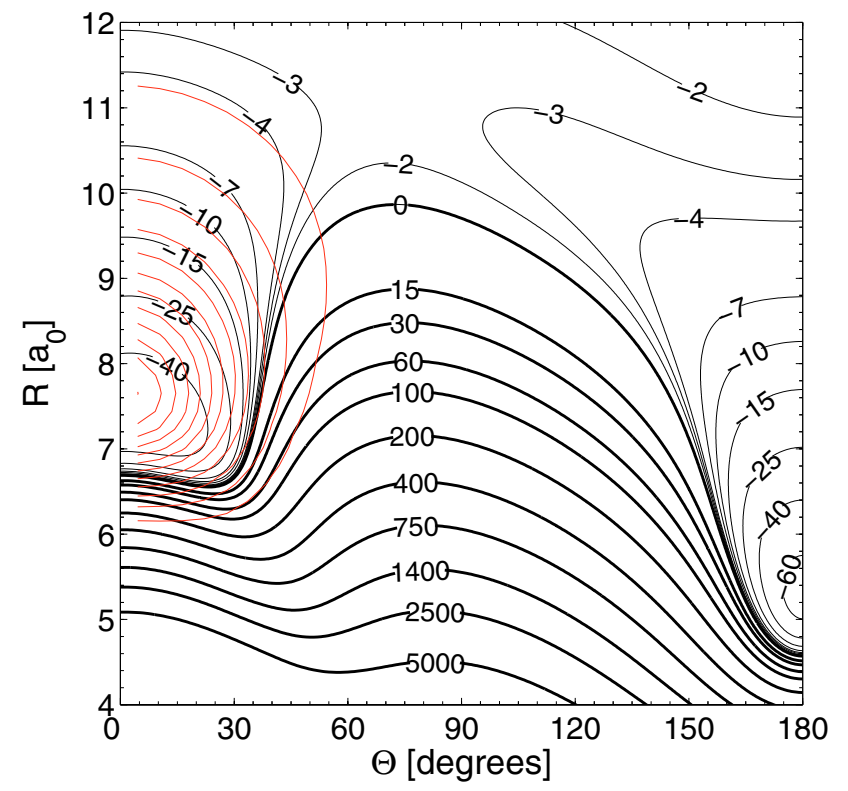

Fig. 1. Contour plot of the PN-He PES as a function of $R$ and $\theta$ with the PN bond distance fixed at $r_{\mathrm{e}}=2.81733$ bohr. The energies are in $\mathrm{cm}^{-1}$ with the zero of energy taken to be $\mathrm{PN}\left(r=r_{\mathrm{e}}\right)+\mathrm{He}$ at infinite separation. The contour lines of the lowest bound state bend-stretch wave function are superimposed on the contour plot of the PES. The ground state wave function is localized on the $\theta=0$ side but extends considerably into the region of T-shaped geometry.

by averaging over the intermolecular ground state vibrational wavefunction are very similar. Consequently, in the present case we anticipate that restricting $r$ to its equilibrium value will introduce little error into the calculated inelastic rate coefficients.

The potential energy surface (PES) was calculated in the supermolecular approach by means of a single- and doubleexcitation coupled-cluster method (Hampel et al. 1993) (CCSD). In addition, we included perturbative contributions from connected triple excitations computed as defined by Watts et al. (1993) $[\operatorname{CCSD}(\mathrm{T})]$. The calculations were done with the MOLPRO 2006.1 package (2002). For all three atoms we used the standard correlation-consistent polarized valence-triple-zeta basis sets of Dunning (1989) (cc-pVTZ) augmented with the diffuse functions of s, p, d, and f symmetries suggested by Kendall (1992) (aug-cc-pVTZ). This basis set was further augmented by the $[3 \mathrm{~s} 3 \mathrm{p} 2 \mathrm{~d} 2 \mathrm{f} 1 \mathrm{~g}]$ bond functions optimized by Cybulski \& Toczyłowski (1999) which were placed midway between the He atom and the PN center of mass. Recently, Lique et al. (2005) have shown that, for weakly bound systems, potential energy surfaces determined with aug-cc-pVTZ basis sets augmented with bond functions are comparable in accuracy to those calculated with larger aug-cc-pVQZ basis sets.

At all geometries the Boys \& Bernardi (1970) counterpoise procedure is used to correct for basis set superposition error (BSSE). In this procedure the interaction energy is defined by

$V(R, \theta)=E_{\mathrm{PN}-\mathrm{He}}(R, \theta)-E_{\mathrm{PN}}(R, \theta)-E_{\mathrm{He}}(R, \theta)$

where the energies of the PN and He subsystems are determined with the full (three atom plus bond function) basis set.

Interaction energies at a total of 455 geometries were computed. The values of the radial scattering coordinate $R$ ranged from 4 bohr to 16 bohr in steps of 0.25 bohr. The angular grid was uniform with a 15 degree spacing from 0 to $180^{\circ}$. At some angles, it was necessary to include smaller values of $R$ to ensure that the calculations extended into the short-range repulsive

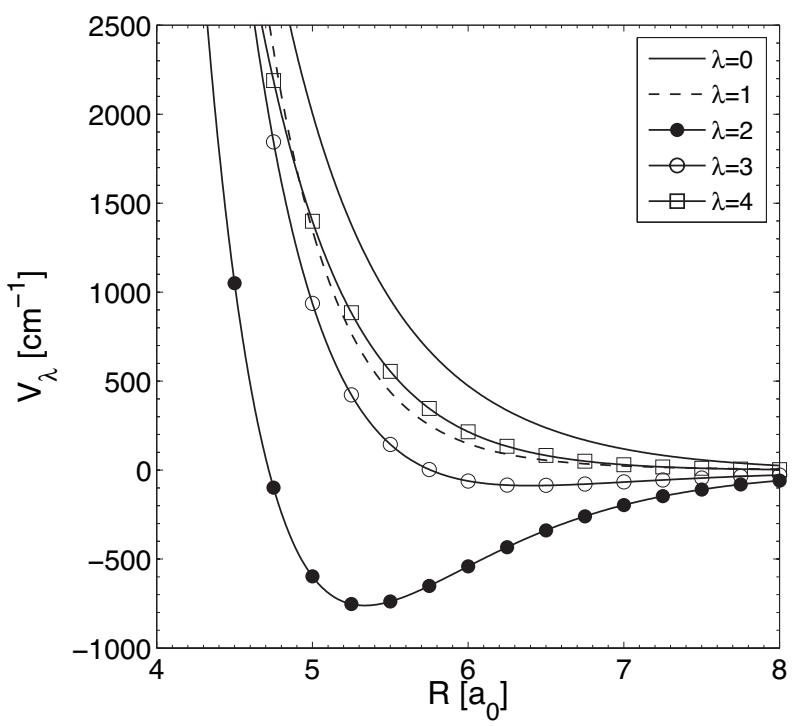

Fig. 2. Plot of first six radial Legendre expansion coefficients $(\lambda=$ $0 . .4)$.

region of the potential. A contour plot of the potential is shown in Fig. 1. For this weakly-bound system the global minimum in the interaction energy was found to be $-67.26 \mathrm{~cm}^{-1}(R=$ 5.32 bohr, $\theta=180^{\circ}$ ) corresponding to collinear He-PN. In HeNP collinear geometry $(\theta=0)$ there exists a local mimimum with well depth $-53.80 \mathrm{~cm}^{-1}$ at a distance of $R=7.38 \mathrm{bohr}$.

The calculated interaction energies were fitted by means of the procedure described by Werner et al. (1988) for the $\mathrm{CN}-\mathrm{He}$ system. The largest deviations between the fit and the ab initio points occur primarily in the repulsive region of the PES. Over the entire grid, the mean relative difference between the analytic fit and the ab initio calculations is $0.5 \%$. The dependence of the potential energy surface on the He-PN angle was fitted by the usual Legendre expansion

$V\left(r=r_{\mathrm{e}}, R, \theta\right)=\sum_{\lambda} V_{\lambda}(R) P_{\lambda}(\cos \theta)$.

From an ab initio grid containing 13 values of $\theta$, we were able to include terms up to $\lambda_{\max }=12$. The dependence on $R$ of the dominant coefficients is shown in Fig. 2. We observe that the largest (in magnitude) of the anisotropic terms $(\lambda>0)$ corresponds to $\lambda=2$. This implies that, to a first approximation, the PES is symmetric with respect to $\theta \rightarrow \pi-\theta$.

We used this fitted potential to determine the bound states of the PN-He system for total angular momentum $J=0$, making use of the collocation method employed previously for the ArHCN system by Cybulski et al. (1999). The calculated dissociation energy, $D_{0}$, is $4.98 \mathrm{~cm}^{-1}$. There exists a second bound state, almost dissociative (bound only by $0.346 \mathrm{~cm}^{-1}$ ). This state corresponds to the first excited mode of the van der Waals stretch.

\section{Collision dynamics}

The main focus of this paper is the use of the fitted PNHe PES to determine rotational excitation and de-excitation cross sections of PN molecules by He atoms. Because the hyperfine structure of the PN molecule is not resolved by current astrophysical spectroscopic observations, we consider only the rotational structure of PN. The rotational energy levels of the ${ }^{31} \mathrm{P}^{14} \mathrm{~N}$ molecule were computed using the experimental spectroscopic constants of Wyse et al. (1973). To determine the inelastic cross sections 
we used the exact close coupling (CC) approach of Arthurs \& Dalgarno (1960).

Because the full $\mathrm{CC}$ approach is very computationally intensive for molecules with small rotational constants, we also used the coupled-states approximation (CS) (McGuire \& Kouri 1974) for the determination of cross sections involving higher rotational levels. In both the CC and CS approach, the integral cross sections are obtained by summing the partial cross sections over a sufficiently large number of values of the total angular momentum $J$ until convergence is reached.

The standard time-independent coupled scattering equations were solved using, for the CC calculations, the HIBRIDON 4 code (Alexander et al. 2004) and, for the CS calculations, the MOLSCAT (Hutson \& Green 1995) code. Calculations were carried out at values of the total energy ranging from 1.8 to $2500 \mathrm{~cm}^{-1}$. The integration parameters were chosen to ensure convergence of the cross sections over this range. In most of the calculations the integration range extended from 3.2 to $40 \mathrm{bohr}$. At the highest collision energy considered $\left(2500 \mathrm{~cm}^{-1}\right)$, the computational expense of the full CC calculations precluded the determination of rotationally inelastic cross sections out of (or into) rotational levels with $j>15$. For transitions involving higher rotational levels $(16<j \leq 30)$ only CS calculations were performed.

The CS approach is expected to give accurate results at high energies and for transitions between high rotational levels for a heavy system like PN. However, errors can arise whenever weak collisions at large impact parameter make a significant contribution, as will occur at low energies or for transitions between closely-spaced levels. These errors will affect, predominately, the calculated rate coefficients only at low kinetic temperatures $(T<50 \mathrm{~K})$. However, for astrophysical modeling at low temperature, transitions involving high rotational levels are unimportant, since these levels are insignificantly populated. Consequently, the CS cross sections involving these high rotational levels will be of importance only over the range of collision energies where the approximation itself is accurate.

For both the CC and CS calculations we extended the rotational basis sufficiently to ensure convergence of the inelastic cross sections for $j, j^{\prime} \leq 15$ (CC) and $j, j^{\prime} \leq 30$ (CS). At the largest total energy considered $\left(2500 \mathrm{~cm}^{-1}\right)$ the rotational basis extending to $j=24$ and 38 , respectively, for the CC and CS calculations. The maximum value of the total angular momentum $J$ used in the calculations was set large enough that the inelastic cross sections were converged to within $0.005 \AA^{2}$.

From the rotationally inelastic cross sections $\sigma_{j \rightarrow j^{\prime}}\left(E_{\mathrm{c}}\right)$, one can obtain the corresponding thermal rate coefficients at temperature $T$ by an average over the collision energy $\left(E_{\mathrm{c}}\right)$ (Smith 1980):

$$
\begin{aligned}
k_{j \rightarrow j^{\prime}}(T)= & \left(\frac{8}{\pi \mu k^{3} T^{3}}\right)^{\frac{1}{2}} \\
& \times \int_{0}^{\infty} \sigma_{j \rightarrow j^{\prime}}\left(E_{\mathrm{c}}\right) E_{\mathrm{c}} \exp \left(-E_{\mathrm{c}} / k T\right) \mathrm{d} E_{\mathrm{c}}
\end{aligned}
$$

where $k$ is Boltzmann's constant. The numerical integration in Eq. (3) was performed using a repeated Simpson's rule. To obtain precise values for the rate constants, the energy grid was chosen to be sufficiently fine to include the numerous scattering resonances which will be described below.

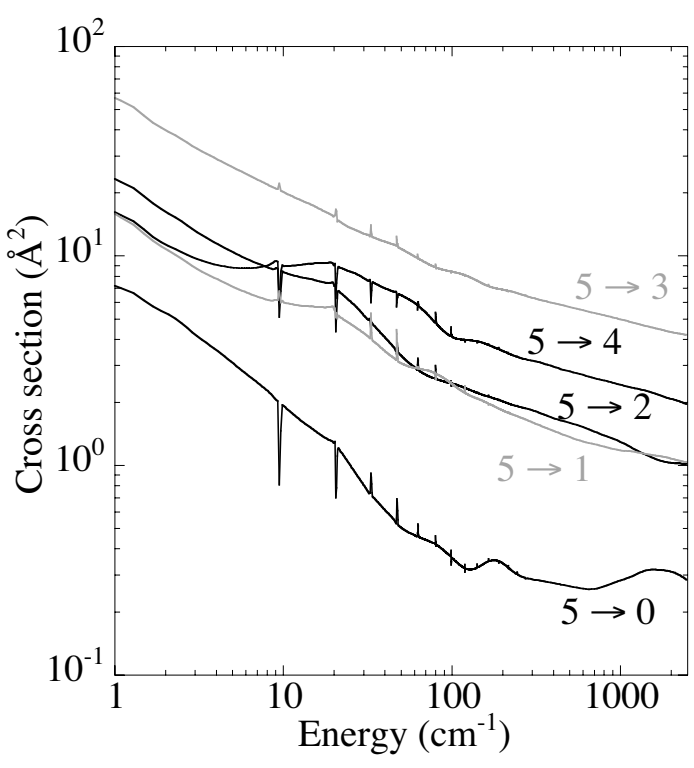

Fig. 3. Typical rotational de-excitation cross sections for the PN molecule in collision with $\mathrm{He}$ as a function of the collision energy. black lines: odd $\Delta j$ transitions; grey lines: even $\Delta j$ transitions.

\section{Results}

\subsection{Cross sections}

Figure 3 illustrates the energy dependence of the collisional deexcitation cross sections obtained from the present $\mathrm{CC}$ calculations for a few selected rotational levels. At collision energies below $\approx 100 \mathrm{~cm}^{-1}$ numerous sharp spikes appear. These are a consequence of the two attractive potential wells (see above) in both linear geometries. Quasibound states may arise from tunneling through the centrifugal energy barrier (shape resonances) or from excitation of the He-PN complex to a bend-stretch level which is energetically accessible because of the attractive wells but is asymptotically closed (Feschbach resonances). Because of the small energy spacing between the PN rotational levels, there are many of these Feschbach resonances. It is the temporary trapping which leads to narrow spikes or dips in the integral cross sections (Smith et al. 1979; Christoffel \& Bowman 1983).

Because of the averaging over collision energy (Eq. (3)) these narrow resonances will have little, if any, effect on the relaxation rate coefficients. However, the strong propensity seen in Fig. 3 in favor of transitions with even $\Delta j$, will persist in the rate coefficients. This propensity is due to the near-homonuclear symmetry of the potential energy surface (see Figs. 1 and 2). This effect has been seen both experimentally (for NO-Ar collisions, Andresen et al. 1982) and theoretically (for CO-He collisions, Chapman \& Green 1977); it was first explained by McCurdy \& Miller (1977).

\subsection{Rate coefficients}

We obtained, by energy averaging, de-excitation rate coefficients for the first $16(j=0-15)$ and next $15(j=16-30)$ rotational levels of PN, from the CC and CS cross sections, respectively. The representative variation with temperature is illustrated in Fig. 4.

As discussed earlier, we expect that at moderate to high collision energy the CC and CS cross sections will be quite similar, especially for transitions among higher rotational levels. This will be similarly true for the corresponding rate coefficients. Figure 5 illustrates the degree of agreement at $T=100^{\circ}$. 

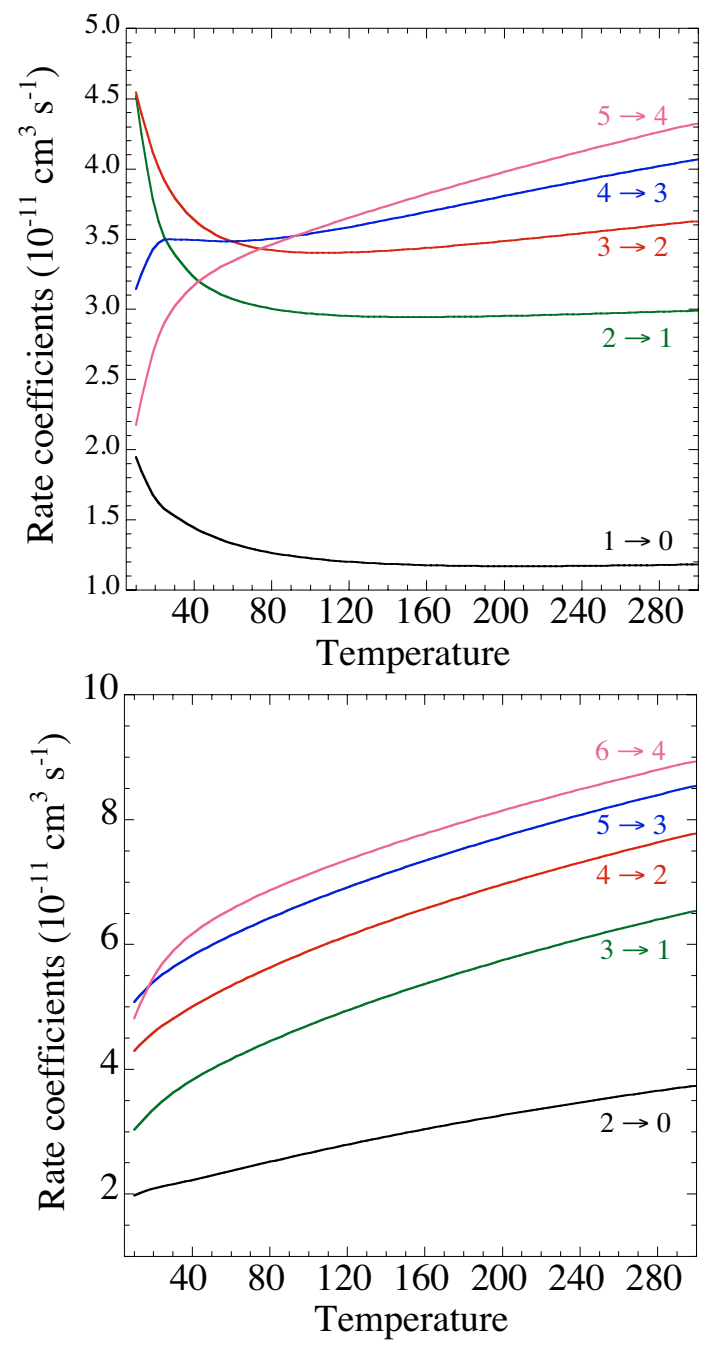

Fig. 4. Typical variation with temperature of collisional de-excitation rate coefficients of $\mathrm{PN}$ by He corresponding to $\Delta j=-1$ (upper panel) and $\Delta j=-2$ (lower panel) transitions.

At lower temperatures (not shown), the degree of agreement deteriorates.

Despite significant differences for transitions involving low rotational levels, the $\mathrm{CC}$ and $\mathrm{CS}$ rate coefficients for transitions involving high rotational levels agree to within $10 \%$. We are confident, then, that the CS rate coefficients for transitions between high rotational levels at $T>100 \mathrm{~K}$ will be quite accurate. The complete set of rotational de-excitation rate coefficients obtained from the CC cross sections for transitions with $j, j^{\prime} \leq 15$ and from the CS cross sections for transitions with $16<j \leq 30$ is expected to be accurate since, as seen previously, for astrophysical modeling transitions implying high rotational levels are not needed at low temperature.

This complete set of (de)excitation rate coefficients is available on-line from the BASECOL website ${ }^{1}$. Excitation rate coefficients can be easily obtained by detailed balance:

$k_{j \rightarrow j^{\prime}}(T)=k_{j^{\prime} \rightarrow j}(T) \frac{2 j^{\prime}+1}{2 j+1} \exp \left[-\left(\varepsilon_{j^{\prime}}-\varepsilon_{j}\right) / k T\right]$

where $\varepsilon_{j}$ and $\varepsilon_{j^{\prime}}$ are, respectively, the energies of the rotational levels $j$ and $j^{\prime}$.

\footnotetext{
${ }^{1}$ http://www.obspm.fr/basecol/
}

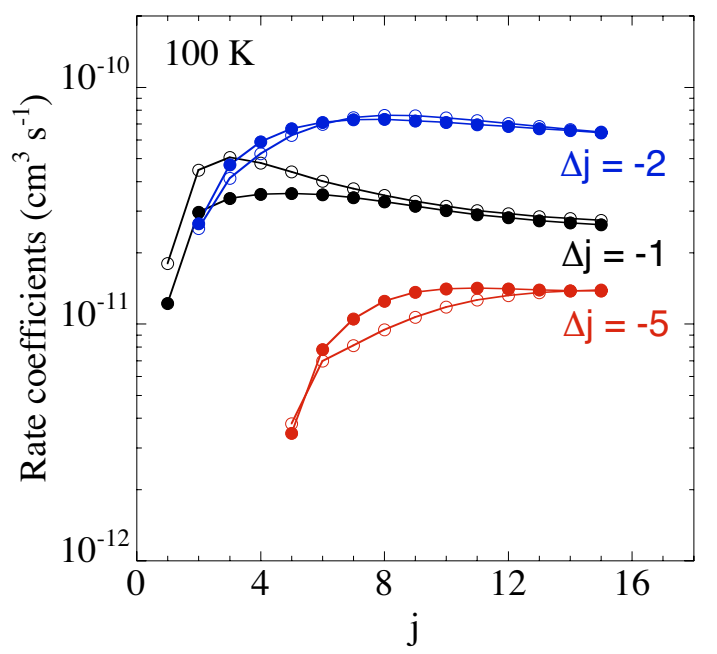

Fig. 5. Comparison between CS (open circles) and CC (filled circles) rate coefficients at $100 \mathrm{~K}$ for $\Delta j=j^{\prime}-j=-1,-2$, and -5 .

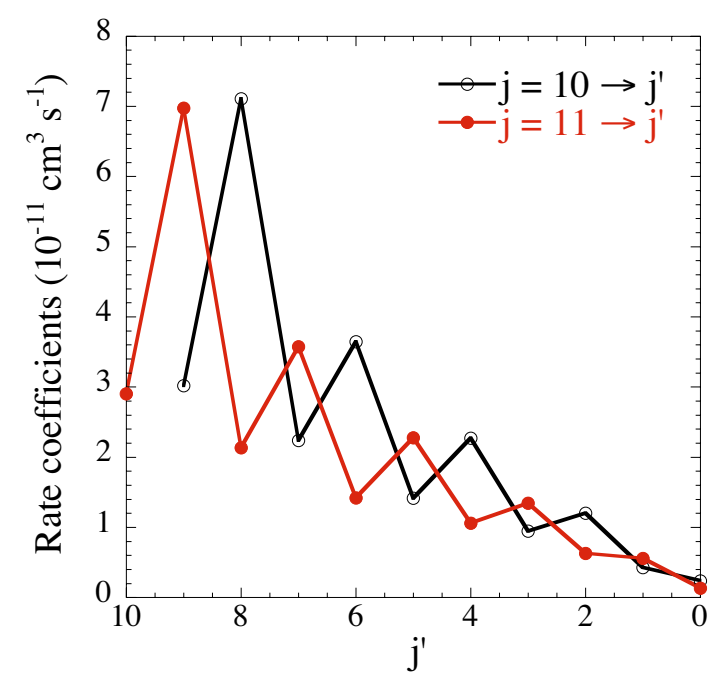

Fig. 6. De-excitation rate coefficients at $100 \mathrm{~K}$ from the $j=10$ and $j=11$ levels.

As anticipated earlier, the rate coefficients display the same propensity toward transitions with even $\Delta j$. This is illustrated by Fig. 6 which shows the de-excitation rate coefficients at $T=$ $100 \mathrm{~K}$ out of the $j=10$ and $j=11$ levels. The de-excitation rate coefficients in Fig. 6 also display the expected overall decrease with increasing $\Delta j$, which is the usual trend.

\section{Summary and discussion}

We have used quantum scattering calculations to investigate rotational energy transfer in collisions of PN with He atoms. The calculations are based on a new, highly accurate 2D potential energy surface. Rate coefficients for transitions involving the lowest 31 levels of this molecule were determined for temperatures ranging from 5 to $300 \mathrm{~K}$. Strong propensity rules for even $\Delta j$ were found. In particular, the rate coefficients for $|\Delta j|=2$ transitions were $\approx 3$ times larger than those for $|\Delta j|=1$ transitions. This strong propensity can have important astrophysical consequences. As was shown by Wernli et al. (2006) for $\mathrm{HC}_{3} \mathrm{~N}$ collisions, a $\Delta j$ even propensity can amplify population inversion if local thermodynamic equilibrium (LTE) conditions are not met. In any case, the full set of rate coefficients presented 
here will enhance our ability to understand and interpret future PN observations.

Finally, the great abundance of $\mathrm{H}_{2}$ in the interstellar medium makes this molecule the primary collision partner for any other species. It is generally assumed (Schöier et al. 2005) that rate coefficients with $\mathrm{He}$ can provide a good estimate of rate coefficients for collision with para- $\mathrm{H}_{2}(j=0)$. This approximation postulates that collisional cross sections with $\mathrm{He}$ and $\operatorname{para}-\mathrm{H}_{2}(j=0)$ are equal, so that the rate coefficients differ only by a reduced mass factor of $\approx 1.4$ arising from the thermal averaging (Eq. (3)). However, some caution must be exercised concerning the use of the present $\mathrm{PN}-\mathrm{He}$ rate coefficients to simulate those for collision of $\mathrm{PN}$ with para- $\mathrm{H}_{2}(j=0)$. Recent results on rotational excitation of $\mathrm{CO}$ (Wernli et al. 2006), $\mathrm{HC}_{3} \mathrm{~N}$ and $\mathrm{SO}$ (Lique et al. 2007) have pointed out that rate coefficients for collisions with para- $\mathrm{H}_{2}(j=0)$ can be up to a factor of 3 larger or smaller than those for collisions with $\mathrm{He}$, depending on the selected transition. Thus, although the present results may provide a good first estimate of collisional rate coefficients for collision of PN with $\mathrm{H}_{2}$, it is crucial to extend the calculations, both of the PES and of the inelastic cross sections, to the $\mathrm{PN}-\mathrm{H}_{2}$ system.

Acknowledgements. The computational component of this work was done using the computer cluster at the Computing Center of the Faculty of Chemistry, Warsaw University. J.K., F.L., and M.H.A. would like to acknowledge financial support from the US National Science Foundation under grant CHE-0413743. G.Ch. acknowledges financial support by the National Science Foundation grant CHE-0414241.

\section{References}

Andresen, P., Joswig, H., Pauly, H., \& Schinke, R. 1982, J. Chem. Phys., 77, 2204

Arthurs, A. M., \& Dalgarno, A. 1960, Proc. Roy. Soc. A, 256, 540

Boys, S. F., \& Bernardi, F. 1970, Mol. Phys., 19, 553

Chapman, S., \& Green, S. 1977, J. Chem. Phys., 67, 2317

Charnley, S. B., \& Millar, T. J. 1994, MNRAS, 270, 570

Christoffel, K. M., \& Bowman, J. M. 1983, J. Chem. Phys., 78, 3952

Cybulski, S. M., \& Toczyłowski, R. R. 1999, J. Chem. Phys., 111, 10520

Cybulski, S. M., Couvillion, J., Kłos, J., \& Chałasiński, G. 1999, J. Chem. Phys., 110,1416

Daniel, F., Cernicharo, J., \& Dubernet, M.-L. 2006, ApJ, 648, 461

Dunning, T. H. 1989, J. Chem. Phys., 90, 1007
Huber, K. P., \& Herzberg, G. 1979, Constants for diatomic molecules, ed. Van Nostrand Reinhold Company

HIBRIDON is a package of programs for the time-independent, quantum treatment of inelastic collisions and photodissociation written by Alexander, M. H., Manolopoulos, D. E., Werner, H.-J., \& Follmeg, B., with contributions by Vohralik, P. F., Lemoine, D., Corey, G., Gordon, R., Johnson, B., Orlikowski, T., Berning, A., Degli-Esposti, A., Rist, C., Dagdigian, P., Pouilly, B., van der Sanden, G., Yang, M., de Weerd, F., Gregurick, S., \& Kłos, J.

Hampel, C., Peterson, K., \& Werner, H.-J. 1992, Chem. Phys. Lett., 190, 1

Hutson, J. M., \& Green, S., MOLSCAT computer code, version 14 (Collaborative Computational Project No. 6 of the Science and Engineering Research Council, UK, 1994)

Kendall, R. A., Dunning, T. H., \& Harrison, R. J. 1992, J. Chem. Phys., 96, 6796 Lique, F., \& Spielfiedel, A. 2007, A\&A, 462, 1179

Lique, F., Spielfiedel, A., Dubernet, M.-L., \& Feautrier, N. 2005, J. Chem. Phys., 123,134316

Lique, F., Cernicharo, J., \& Cox, P. 2006, ApJ, 653, 1342

Lique, F., Senent, M.-L., Spielfiedel, A., \& Feautrier, N. 2007, J. Chem. Phys., 126,164312

Manolopoulos, D. E. 1986, J. Chem. Phys., 85, 6425

McCurdy, C. W., \& Miller, W. H. 1977, J. Chem. Phys., 67, 463

McGuire, P., \& Kouri, D. J. 1974, J. Chem. Phys., 60, 2488

Millar, T. J. 1994, A\&A, 42, 241

MOLPRO is a package of ab initio programs written by: Werner, H.-J., \& Knowles, P. J. with contributions from Almlöf, J., Amos, R. D., Deegan, M. J., Elbert, S. T., Hampel, C., Meyer, W., Peterson, K., Pitzer, R., Stone, A. J., Taylor, P. R., Lindh, R., Mura, M. E., \& Thorsteisson, T.

Schilke, P., Groesbeck, T. D., Blake, G. A., \& Phillips, T. G. 1997, ApJS, 108, 301

Schöier, F. L., van der Tak, F. F. S., van Dishoeck, E. F., \& Black, J. H. 2005, A\&A, 432, 369

Smith, I. W. M., Kinetics, \& dynamics of elementary gas reactions 1980, ed. Butterworths, London

Smith, L. N., Malik, D. J., \& Secrest, D. 1979, J. Chem. Phys., 71, 4502

Tercero, B., \& Cernicharo, J. 2007, to be published

Turner, B. E. 1987, A\&A, 183, L23

Turner, B. E. 1991, ApJ, 376, 573

Turner, B. E., \& Bally, J. 1987, ApJ, 321, L75

Turner, B. E., Tsuji, T., Bally, J., Guelin, M., \& Cernicharo, J. 1990, ApJ, 365, 569

Turner, B. E., Chan, K.-W., Green, S., \& Lubowich, D. A. 1992, ApJ, 399, 114

Watts, J. D., Gauss, J., \& Bartlett, R. J. 1993, J. Chem. Phys., 98, 8718

Werner, H.-J., Follmeg, B., \& Alexander, M. H. 1988, J. Chem. Phys., 89, 3139

Wernli, M., Valiron, P., Faure, A., et al. 2006, A\&A, 446, 367

Wernli, M., Wiesenfeld, L., Faure, A., \& Valiron, P. 2007, A\&A, 464, 1147

Wyse, F. C., Manson, E. L., \& Gordy, W. 1972, J. Chem. Phys., 57, 1106

Ziurys, L. M. 1987, ApJ, 321, L81 